\section{Procesos de expansión y límites a la privatización de la atención de la salud en América Latina: más allá de las tipologías}

\author{
Processes of expansion and limits to privatization \\ of healthcare in Latin America: beyond typologies
}

\author{
Processos de expansão e limites à privatização \\ da atenção à saúde na América Latina: além \\ das tipologias
}

Maria José Luzuriaga 1,2

Lígia Bahia 2

doi: 10.1590/0102-311X00127616

\section{Resumen}

El objetivo fue realizar una revisión bibliográfica sobre los sistemas de salud $y$ estudios comparados en politicas y sistemas de salud para identificar los principales aportes de los mismos en el análisis de la privatización, especialmente, el papel de las organizaciones que comercializan planes y seguros de salud en los sistemas nacionales de salud en países de América Latina. La revisión se centró en analizar la adecuación de perspectivas o propuestas teórico-metodológicas y de sus indicadores para dimensionar la participación del sector privado, y su interacción con los sistemas nacionales de salud. De este modo, buscamos problematizar la adecuación, los límites y las potencialidades de los mismos, en el estudio de los procesos de privatización en los sistemas de salud de países de América Latina. El análisis comparado de algunos elementos específicos, como el análisis de gastos, la capacidad instalada, la población cubierta con planes y seguros privados en los sistema de salud, nos permitió identificar matices, contradicciones existentes en los sistemas, respecto a la relación público-privada, así como cuestionarnos el uso de clasificaciones tradicionales con las que se definieron diversos sistemas de salud en nuestra región. Nuestro desafío fue romper con algunas explicaciones lineales o ausentes de contradicciones sobre los análisis de los sistemas de salud. Desde esa perspectiva, es necesario cuestionar categorías o tipologías que se han desarrollado y utilizado de manera recurrente, con el fin de que se discutan y repiensen para poder avanzar en una comprensión más rigurosa de nuestras realidades, así como estimular nuevos debates y explicaciones.

Privatización; Sistemas Prepagos de Salud; Sistemas de Salud; Politica de Salud; América Latina
Correspondência

M. J. Luzuriaga

Instituto de Salud Colectiva, Universidad Nacional de Lanús. 30 de Septiembre, Adrogué, Buenos Aires 1846, Argentina. mariajoseluzuriaga@gmail.com

\footnotetext{
1 Instituto de Salud Colectiva, Universidad Nacional de Lanús, Buenos Aires, Argentina.

2 Instituto de Estudos em Saúde Coletiva, Universidade Federal do Rio de Janeiro, Rio de Janeiro, Brasil.
} 


\section{Introducción}

Al realizar un recorrido por los estudios comparados que se han desarrollado en el campo de la salud, es posible identificar un predominio de aquellos elaborados bajo un abordaje meramente pragmático sobre sistemas de salud realizados en los años sesenta y setenta. También se observa una gran producción de libros (guías) de orientación para comparar sistemas de salud, en su mayoría inspirados en el trabajo de Talcott Parsons 1. Otra vertiente de investigaciones, basadas en la comparación entre países, se apoya en la cuantificación de la percepción sobre el estatus de salud y el uso de servicios, ejemplificada por el estudio clásico sobre las diferencias en la percepción sobre enfermedades y uso de servicios de salud 2. Por otra parte, un estudio de referencia es el trabajo titulado Health Care Systems in the World de Milton Roemer ${ }^{3}$, que si bien ha sido criticado por basarse en una perspectiva acrítica 4 fue ampliamente difundido. A partir de los años 1990, las reflexiones sobre los sistemas comparados de salud se concentraron en estudios econométricos, centrados en el financiamiento y la equidad, dedicados a evidenciar la mayor efectividad de los sistemas de salud europeos universales, en contraste con el sistema de Estados Unidos 5.

Marmor \& Wendt 6 realizaron una revisión de la producción académica, con el fin de identificar los marcos conceptuales que han sido utilizados por los estudios comparativos en políticas y sistemas de salud y en los mismos identificaron tres problemas predominantes: la falta del conocimiento de los contextos locales donde se implementan las políticas; la falta de análisis de los resultados de las políticas; y la falta de atención en los estudios sobre actores políticos involucrados en los procesos políticos. En relación con las potencialidades de los estudios comparados, destacan la capacidad de los mismos para un mayor conocimiento de las experiencias propias, así como la posibilidad de una lectura de un rango más amplio y con explicaciones que superen el denominado "provincianismo", o sea, explicaciones basadas en aspectos muy locales 7 . De esta manera, el primer elemento positivo de la investigación transnacional es la posibilidad de comprender en profundidad las propias circunstancias a partir de la comparación.

El conocimiento sobre los sistemas de otros países ofrece lecciones en perspectiva, no directas. Ilumina diferencias sutiles entre naciones que son muy similares y las diferencias más nítidas cuando se aplica a regímenes nacionales diferentes. Podríamos denominar esta comparación "iluminación sin trasplante de la política" 7 .

Para comprender el contexto político más amplio, en el cual se dan los debates de la política de salud, se sugiere considerar cuatro reglas básicas: (1) la necesidad de reflexionar sobre el verdadero objetivo de realizar comparaciones; (2) realizar una reflexión explícita sobre la situación del sistema de salud en los sistemas de protección social modernos, teniendo en cuenta las responsabilidades de lo público y lo privado, en cuanto a financiación, contratación y propiedad de los servicios de salud; (3) elaborar definiciones precisas y operacionales para los debates de las políticas, en vez de etiquetas engañosas de marketing (recomendaciones particularmente relevantes en la discusión de "modelos" para organizar y financiar la atención de la salud); (4) la necesidad de comprender la constelación de valores dominantes del país específico, las instituciones políticas y el papel de los intereses organizados en el ámbito de la salud 8,9 .

También se propone y recomienda que en el momento de estudiar sistemas de salud es preferible comparar políticas o programas antes que sistemas de países en su totalidad, ya que "es más fácil categorizar (o caracterizar) programas y politicas que configuraciones enteras de salud de los países. Este nivel de análisis ofrece un laboratorio más realista de cambio" 10 (p. 212).

El objetivo del presente trabajo fue realizar una revisión bibliográfica sobre estudios comparados, en políticas y sistemas de salud, para identificar los principales aportes de los mismos, en el análisis de la privatización, especialmente, el papel de las organizaciones que comercializan planes y seguros de salud en países de América Latina. Para ello fueron consideradas dos vertientes analíticas. Por un lado, la literatura internacional, que se ha centrado en comprender la dinámica de las organizaciones que comercializan planes y seguros de salud y, por otro lado, la producción de estudios dedicados al análisis de los sistemas de salud, desde una perspectiva comparada, y preocupados en el desarrollo de indicadores capaces de dimensionar la participación del sector privado y/o la relación público/privada en el sistema de salud. De esta manera, nos propusimos cuestionar algunas categorías y modelos con los que son asociados los sistemas de salud de diversos países de América Latina. Consideramos 
que las interpretaciones sobre la privatización, y sus desdoblamientos conceptuales y pragmáticos, contribuyen a la problematización sobre la adhesión a la realidad de determinados modelos de clasificación en países de América del Sur.

En la región encontramos referencias importantes en el desarrollo de estudios comparados que, en su mayoría, han utilizado una perspectiva histórica con énfasis en los cambios institucionales de los sistemas de salud 11,12,13,14,15,16,17,18,19. En los mismos se pueden identificar tanto las semejanzas como las diferencias entre las trayectorias a partir del análisis de los actores sociales involucrados y los conflictos de interés en juego 11,13,15,17; los contextos políticos y económicos que han favorecido procesos de reforma 14,15,16,17,18; el rol de los organismos internacionales 17,18,19; los procesos de implementación de las reformas y los resultados en términos de igualdad y universalidad de acceso 13,14,16,18, entre otros. Estos trabajos han sido de gran relevancia en el debate acerca de las políticas y sistemas de salud en nuestra región, particularmente en la producción académica. Sin embargo, cuando nos enfocamos en el estudio de los procesos de privatización se observan vacíos, en relación a la comprensión de las políticas de privatización en los análisis mencionados. Ciertamente, el foco de estos estudios se dirigió a la comprensión de los procesos de reforma, y no a la búsqueda de la comprensión de las múltiples interacciones de los componentes públicos y privados de los sistemas de salud. Por otra parte, la recurrencia en la utilización de ciertas tipologías 20,21, con las que se han asociado algunos de los sistemas de salud de los países de nuestra región, como el caso de Chile como sistema dual, Colombia, como de pluralismo estructurado 20, y Brasil como sistema universal, ha impedido una profundización en la búsqueda de las contradicciones y tensiones subyacentes en los arreglos instituciones y, particularmente, en la búsqueda de reversión de los procesos que limitan la implementación de políticas universales e igualitarias.

El trabajo tiene como hilo conductor la revisión bibliográfica estructurada en dos vertientes. La primera concentrada en la búsqueda intencional en la literatura regional e internacional de bases conceptuales para subsidiar una reflexión centrada en la evaluación de los contextos singulares, el origen de las políticas públicas privatizantes, la implementación de las normas legales y sus resultados. En dicha literatura predomina una comprensión crítica del análisis comparativo y de los procesos de privatización. La segunda vertiente de la revisión se conformó, a partir de la consulta de trabajos de investigadores latinoamericanos sobre reformas y sistemas de salud, considerando aquellos más citados. Por último, la revisión fue complementada con el revelamiento de datos secundarios sobre Argentina, Brasil, Chile y Colombia. La elección de los casos fue motivada por la fuerte presencia del componente privado, así como por la singular participación en cada uno de los sistemas de salud de los países estudiados.

\section{Privatización: concepciones para el análisis de políticas de salud}

Un consenso que se destaca en diversos trabajos sobre la privatización es la gran confusión que ha generado la necesidad de designar una configuración determinada de un sistema de salud, como predominantemente pública o privada, siendo que en esta clasificación el componente ideológico 22,23,24 ha predominando sobre los análisis que proponen una conceptualización del componente privado, y en base a qué evidencias empíricas fundamentan sus afirmaciones. En ese sentido, el primer elemento que se destaca en los trabajos citados 22,23,24 es el esfuerzo por definir con precisión qué se entiende por público y por privado, y en qué casos puede observarse un proceso de privatización. A su vez, también se destaca la necesidad de buscar marcos que permitan identificar las nuevas configuraciones en las que las fronteras entre lo público y lo privado se hacen cada vez más obsoletas y debe pensarse en un continuum o lecturas menos estáticas.

Paul Starr propone un análisis minucioso de lo que implica el concepto privatización, identificando las principales causas que provocan confusión en el debate en torno al tema, los límites en el tratamiento del mismo por algunas perspectivas teóricas y, finalmente, plantea una serie de dimensiones para poder aproximarnos a un análisis más adecuado y preciso 22 . El autor estructura el trabajo, a partir de una consideración central subdividida en cuatro ejes: idea, teoría, retórica y como práctica política. En relación a la comprensión de la privatización como una idea, destaca por un lado, la complejidad que implica la definición de los límites entre lo público y lo privado, y por otro lado, el 
significado político de la misma. Otro aspecto central que el autor destaca es que existen diferentes grados en los que el proceso puede desarrollarse, y el grado en el que se lleva a cabo es central para evaluar las posibles consecuencias, en relación al impacto social de dichas políticas. En ese sentido, pone énfasis en identificar el grado en el que se mueve la propiedad, las finanzas y la responsabilidad del sector público 22 .

En una perspectiva de análisis y de preocupaciones teóricas y metodológicas similar a la de Starr encontramos el trabajo de Maarse 23. El supuesto que se sostiene es que existen múltiples fronteras en la relación público/privada en la atención de la salud. Una de sus propuestas se centra en la necesidad de incorporar el carácter evolutivo del proceso, así como identificar los matices que existen en la relación público/privada para comprender el proceso de privatización. Para tal fin, propone analizar el proceso de privatización "como una secuencia continua de recorridos" 23 (p. 988). Otro elemento clave de su propuesta teórica es un enfoque pragmático para investigar la privatización, a partir de cuatro perspectivas diferentes: "la financiación de la atención de la salud, la provisión de la atención de la salud, la administración y gestión y, finalmente, la inversión de la atención de la salud" 23 (p. 986). El estudio se centró en identificar qué factores han empujado la privatización y cuáles han actuado como barreras a la misma. Los resultados expuestos plantean que en los países estudiados "hay evidencia de que la atención de la salud en Europa se ha convertido en algo más privado” 23 (p. 981). Lo fundamental, entre otros aspectos, se encuentra en que el crecimiento de la fracción pública en el gasto en la atención de la salud se ha estancado desde la década de 1980 y la fracción privada ha aumentado sustantivamente 23. Entre los factores que estimularon la privatización identifica: ideas neoliberales, estancamiento del gasto en el presupuesto público de salud, baja calidad del sector público, y procesos políticos y sociales más amplios. En cuanto a aquellos factores que actuaron como barrera a la privatización encuentra: el acceso universal a los servicios de salud, los valores existentes en la sociedad, estructuras corporativas de gobierno (relaciones entre los diversos agentes que generan una cultura de ajuste mutuo por búsqueda de consensos y compromisos políticos), y fallas del sector privado 23 .

Saltman 24 realiza una propuesta teórica, a partir de la cual poder comprender los límites cada vez menos definidos que existen entre lo público y lo privado, en los sistemas de salud de los países europeos. Destaca algunas concepciones erróneas acerca de la privatización, tales como asociar a la misma con la introducción de mayor competencia, con procesos de autonomización y de corporativización. Refuta la idea de asociar el comportamiento competitivo a la privatización, ya que existen empresas públicas altamente competitivas. Destaca que existen numerosos ejemplos de grandes corporaciones de propiedad privada que son esencialmente monopólicas u oligopólicas, como ejemplos de empresas públicas que pueden competir fuertemente en el mercado. Su propuesta teórica busca superar la dicotomía público/privada. Ubica dentro de la categoría pública dos categorías generales: Estado y otros de propiedad pública, pero no controlada por el Estado; mientras en la categoría privado sitúan organizaciones sin fines de lucro, así como con fines de lucro.

Tras analizar estas relaciones en diversos sistemas de salud como Suecia, Inglaterra, Alemania y España, Saltman 24 observa que estas nuevas configuraciones, en las que los componentes públicos y privados se encuentran más difusos, ya no pueden ser pensadas como anomalías. En cuanto a las principales conclusiones del trabajo, la autora destaca que "la noción de 'público' es cada vez más variada y difusa, y la noción de lo que es 'privado' está en un proceso similar en este respecto” 24 (p. 27). Finalmente, la autora postula la importancia del papel del Estado para un control riguroso, con fuertes medidas restrictivas de estos nuevos arreglos público/privados, de modo de que se impida poner en riesgo los objetivos clínicos y sociales que deben ser priorizados en los sistemas de salud.

Otro trabajo que incorporamos en nuestra propuesta de análisis es el de Bambra 25, quien toma como referencia la propuesta de Esping-Andersen 26 sobre la desmercantilización en su estudio de los regímenes de Estado de Bienestar, y desarrolla el índice de desmercantilización de la salud -The Health Decommodification Index - 25. El índice de desmercantilización lo construye a partir de los datos del gasto privado en salud como porcentaje del Producto Bruto Interno (PBI), las camas de hospitales privados, como porcentaje del total de camas existentes y el porcentaje de la población cubierta por el sistema público de salud 25. La autora sostiene que: "Estos factores fueron elegidos porque evalúan la financiación, la provisión y la cobertura del sector privado y son, por lo tanto, indicadores útiles del rol variado del mercado en el sistema de salud: la amplitud del tamaño del sector privado, en términos de gasto y consumo, el mayor rol del mercado y, por lo tanto, el menor grado de desmercantilización de la salud" 25 (p. 34). 


\section{Privatización y sistemas de salud en América Latina}

En los estudios comparados sobre sistemas de salud realizados en América Latina, realizados a final del siglo XIX e inicios de los años 2000, predominó la clasificación por modelos, por la tendencia considerada predominante de organización de la oferta y la demanda de acciones de salud. De esta manera, el énfasis recayó tanto en una división entre sistemas tipo sistema nacional de salud o seguro social, así como en la identificación de la dualidad resultante de la segmentación de las demandas, mediante la combinación del seguro social y oferta pública, y una variación o predominio de seguros privados, subsidiados por fondos públicos, y la preservación de seguros sociales. Recientemente, con la controvertida propuesta de cobertura universal los debates tienden a concentrarse en torno de la dicotomía entre sistemas orientados por la demanda o por la oferta 27 . Son perspectivas analíticas distintas. El énfasis en el delineamiento de modelos se fundamenta en la comprensión de las estructuras o en las funciones de financiamiento, gestión y prestación de servicios. Mientras que para la evaluación del progreso de las coberturas universales el énfasis recae sobre los avances en la vinculación de segmentos poblaciones a seguros. Sin embargo, la presencia de seguros, sea como indicador de procesos de reformas parciales, sea como implementación de dispositivos para reducir gastos catastróficos de salud ha sido un trazo común en los análisis de ambos enfoques.

Pocas investigaciones utilizaron la privatización como una de las claves para la comprensión de los sistemas y políticas de salud de países de América Latina 18,28. Bahia en uno de sus trabajos propone un modelo explicativo, capaz de superar los análisis de fuerte tenor prescriptivo, y dirigido a evidenciar las tensiones y contradicciones que subyacen en las políticas de salud 29, como clave para la comprensión los límites y perspectivas del Sistema Único de Salud (SUS). En este sentido, su objetivo fue profundizar en el conocimiento sobre la relación entre la base material y la generación de políticas de salud para poder identificar las tendencias de crecimiento/retracción actual de los componentes públicos y privados en el sistema de salud brasileño. Su análisis sobre diversos aspectos teóricos y la dinámica de las políticas de salud resultan útiles para comprender y visibilizar las tensiones y contradicciones existentes en los procesos de privatización en los sistemas de salud de otros países de la región. Una primera reflexión es sobre la falacia de entender el sistema público y privado como realidades autónomas y sin puntos de contacto en sus dinámicas. Como señala la autora: "Es exactamente el desciframiento del complejo proceso de complementariedad público-privada lo que posibilita la elaboración de análisis más realistas de cambios en las bases de la producción de representación de intereses del sistema de salud" 29 (p. 756).

A partir de este planteamiento, Bahia "propone considerar los patrones y los cambios en la división y superposición de ámbitos de actuación y las correspondencias entre segmentación estratificada de la oferta y la demanda" 29 (p. 756). En este análisis identifica dos dimensiones relevantes de flujos financieros asistenciales públicos-privados: una referida a las conexiones entre financiación, oferta y demanda de servicios públicos y privados; y la otra, referida a "los efectos del volumen de los recursos y flujos financieros-asistenciales público/privados, en las bases materiales del trabajo involucrado con la producción de los servicios de salud" 29 (p. 756).

Un estudio comparado sobre los procesos de privatización de los sistemas de salud en Chile, Colombia y México 30 se basó en el marco referencial del estudio de Maarse ${ }^{23}$ para analizar el recorrido de los procesos de privatización en cada uno de los mismos, destacando las divergencias y las convergencias en dicho proceso. Sostienen que la relación público/privada fue semejante en estos países hasta inicios de los años 1980 y luego siguieron trayectorias divergentes 30. Al igual que lo desarrolla Maarse en el trabajo expuesto 23, se mencionan los factores que promueven la privatización y el conjunto de factores que la han limitado. Entre los factores que promovieron la privatización se encuentran: grupos tecnocráticos; contextos políticos específicos; difusión de políticas; privatización impulsada por la demanda; y economía informal. Y en relación a los factores que actuaron como limitantes de este proceso señalan: la movilización de la oposición; las prioridades políticas en competencia; la debilidad institucional; y los sistemas de seguros universales 30 . 


\section{Sobre las organizaciones que comercializan planes y seguros de salud}

La OCDE (Organización para la Cooperación y el Desarrollo Económicos) define al seguro privado de salud como: "Una forma de distribuir el riesgo financiero asociado a la variación de los gastos de atención de salud de la persona, reuniendo en un fondo común los costos en el tiempo (prepago) y entre las personas. En esencia, un seguro de salud privado implica la cobertura de un conjunto de servicios de salud financiados a través de pagos privados, en forma de una prima a un asegurador definido. El asegurador, una entidad no gubernamental, asume gran parte o todo el riesgo de pagar por esos servicios" 31 (p. 3).

Bahia 32 (p. 67) postula la importancia de superar la clasificación dicotómica entre seguros privados y sociales, a partir de características aisladas que los dicotomizan: "Las instituciones privadas estarían subordinadas a las disposiciones del mercado (...) y las públicas a las disposiciones del Estado”. Sin embargo, existen aspectos colectivos y obligatorios en las operaciones de los planes privados que no estarían siendo evidenciados desde una clasificación dicotómica.

Una de las tipologías predominantes de los seguros privados es la propuesta por la OCDE, que se realiza en función del grado de interacción con los sistemas universales de salud, y en ese sentido pueden ser clasificados como complementarios, suplementarios o duplicados 33. Una propuesta diferente la identificamos en el trabajo de Kiil 34, quien tuvo como objetivo revisar la producción de estudios empíricos sobre las características de los seguros privados de salud voluntarios, y evaluar en qué medida la evidencia empírica se corresponde con las predicciones teóricas. Uno de los propósitos principales del análisis se centró en poder evaluar las implicaciones de los mismos sobre la equidad del sistema de salud. En cuanto a los principales resultados, se observó que en concordancia con la teoría económica, la probabilidad de sacar un seguro aumenta con el nivel de ingresos y de educación. También se identificó una asociación positiva en relación al uso de servicios de salud en el pasado y la probabilidad de comprar un seguro privado de salud. En la misma línea, también se observó una relación positiva entre la existencia de listas de espera para realizar consultas y el tiempo para la realización de tratamientos en los sistemas universales de salud y la probabilidad de adquirir un seguro privado de salud. En relación con la autoevaluación de la salud, específicamente respecto a la existencia de alguna enfermedad crónica, no se observó como variable explicativa para la adquisición de seguros de salud en gran parte de los países que fueron analizados 34 .

\section{La diversidad de lo privado: límites de las tipologías de los sistemas de salud}

Documentos oficiales y una parte considerable de la producción técnico-científica describen los sistemas de salud de países de América del Sur, a partir de categorías que reafirman tipologías, especialmente aquellas basadas en dicotomías como unitario/dual, integrado/fragmentado, público/privado, público o privado. La ruptura de las díadas ocurre cuando se toma en consideración el origen de las demandas, a partir de las cuales deriva la inclusión de una tercera categoría como la de obra social, especialmente para designar la existencia de tres sectores (público, privado y seguro social) en el caso de Argentina 35,36.

A su vez, la contraposición adquiere otras clasificaciones cuando están basadas en las fuentes de financiamiento en Chile (Instituciones de Salud Previsional -ISAPRES- y Fondo Nacional de Salud -FONASA) y en Colombia (Régimen Contributivo y Régimen Subsidiado) 37. Para los países cuyos sistemas son clasificados como público y privados, tales conceptos designan fenómenos similares, pero no idénticos. Los planes privados voluntarios de salud en Brasil y los de medicina prepaga en Argentina suelen ser tomados como sinónimo de privado, aunque los primeros tengan parte de su financiación y red asistencial vinculados al sector público 37 y existan, aunque sean residuales, planes privados en Argentina, contratados directamente sin intermediación de un seguro social 38.

En Chile y en Colombia la categoría privado se refiere a planes de salud financiados por contribuciones sociales obligatorias. Los seguros sociales, cuyos fondos son dirigidos a empresas privadas de planes de salud. El término subsidio, especialmente en su forma adjetivada de subsidiado, también define situaciones heterogéneas. En Colombia, se califica el financiamiento público directamente asignado para la cobertura de segmentos poblacionales excluidos del mercado formal de trabajo y en 
Brasil alude a las exenciones y deducciones fiscales para quien paga planes privados en los estratos de mayores ingresos.

Considerando que los rótulos usuales son poco apropiados para la comprensión de la inserción de los planes privados de salud en cada país y la perspectiva de adecuar indicadores de privatización a sistemas de salud de América del Sur se verifica que, si bien los valores del PBI per cápita son más elevados en Chile y en Argentina (Tabla 1), el país que presentó la mayor proporción de gastos públicos como proporción del PBI, en todos los años señalados, en el intervalo de 2000 a 2015, fue Colombia (Tabla 2).

Esa tendencia, de elevada proporción de gastos públicos en relación al total de gastos con salud en Colombia es una excepción. Chile, Brasil y Argentina exhiben altos porcentajes de gastos privados en salud, si bien es plausible admitir, especialmente para Chile, un movimiento discreto de ampliación relativa de gastos públicos. El peso de los gastos en planes privados como proporción del gasto privado fue mayor en Brasil y menor en Argentina y tendieron a aumentar y disminuir respectivamente a lo largo del período analizado (Tabla 3).

Los acercamientos y distanciamientos al público y al privado también son cuestionados por la información sobre oferta de camas y la magnitud de las coberturas de planes privados de salud. En Chile y en Argentina predominan las camas hospitalarias públicas, mientras que en Brasil y Colombia las privadas (Tabla 4). Las coberturas por planes de salud llegaron a alcanzar cerca del $40 \%$ de la población, dentro del Sistema General de Seguridad Social de Salud de Colombia en 2000 y casi el 48\% en 2014; siendo en Brasil el 28\% en 2014 (Tabla 5).

\section{Tabla 1}

Producto Bruto Interno (PBI) per cápita * y gasto público en salud per cápita (en \$ ppp). Argentina, Brasil, Chile y Colombia, 2000, 2005, 2010, 2014 y 2015.

\begin{tabular}{|c|c|c|c|c|c|c|c|c|c|}
\hline \multirow[t]{2}{*}{ Países } & \multicolumn{2}{|c|}{2000} & \multicolumn{2}{|c|}{2005} & \multicolumn{2}{|c|}{2010} & \multicolumn{2}{|c|}{2014} & \multirow{2}{*}{$\begin{array}{c}2015 \\
\text { PBI }\end{array}$} \\
\hline & PBI & $\begin{array}{c}\text { Gasto } \\
\text { público } \\
\text { en salud }\end{array}$ & PBI & $\begin{array}{c}\text { Gasto } \\
\text { público } \\
\text { en salud }\end{array}$ & PBI & $\begin{array}{c}\text { Gasto } \\
\text { público } \\
\text { en salud }\end{array}$ & PBI & $\begin{array}{c}\text { Gasto } \\
\text { público } \\
\text { en salud }\end{array}$ & \\
\hline Argentina & [11.829] & 451 & [13.983] & 477 & [19.520] & 816 & & 630 & [15.168] \\
\hline Brasil & 8.500 [9.108] & 255 & $10.260[11.055]$ & 374 & $13.520[14.340]$ & 534 & 14.750 & 607 & [13.765] \\
\hline Chile & 9320 [11.375] & 320 & $11.700[15.083]$ & 323 & $17.010[18.904]$ & 602 & 21.030 & 865 & [22.850] \\
\hline Colombia & 6520 [6.621] & 308 & 7.970 [8.348] & 356 & $10.130[10.805]$ & 531 & 11.890 & 723 & [12.799] \\
\hline
\end{tabular}

* Los datos entre corchetes tienen como fuente el Fondo Monetario Internacional y, los demás, la Organización Mundial de la Salud, que no dispone de información sobre el PBI per cápita en \$ ppp para Argentina.

Fuentes: elaboración propia, a partir de datos de la Organización Mundial de la Salud (Global Health Observatory data repository. Health expenditure per capita, by country, 1995-2014. (http://apps.who.int/gho/data/node.main.484?lang=en, accedido el Feb/2016) y del Fondo Monetario Internacional (Economic statistics database. http://www.economywatch.com/economic-statistics/economic-indicators/GDP_Per_Capita_PPP, accedido el Feb/2016).

\section{Tabla 2}

Distribución temporal del gasto público en salud como \% del Producto Bruto Interno (PBI). Argentina, Brasil, Chile y Colombia, $2000-2014$.

\begin{tabular}{|c|c|c|c|c|c|c|c|c|c|c|c|c|c|c|c|}
\hline Países & 2000 & 2001 & 2002 & 2003 & 2004 & 2005 & 2006 & 2007 & 2008 & 2009 & 2010 & 2011 & 2012 & 2013 & 2014 \\
\hline Argentina & 5,0 & 5,1 & 4,5 & 4,3 & 3,5 & 3,7 & 3,7 & 3,8 & 4,1 & 5,0 & 4,2 & 3,7 & 3,0 & 2,7 & 2,7 \\
\hline Brasil & 2,8 & 3,0 & 3,2 & 3,1 & 3,3 & 3,4 & 3,5 & 3,5 & 3,6 & 3,8 & 3,8 & 3,7 & 3,7 & 3,8 & 3,8 \\
\hline Chile & 3,3 & 3,5 & 3,5 & 2,7 & 2,6 & 2,5 & 2,5 & 2,7 & 2,9 & 3,5 & 3,3 & 3,3 & 3,5 & 3,6 & 3,9 \\
\hline Colombia & 4,7 & 4,7 & 4,5 & 4,9 & 4,1 & 4,3 & 4,5 & 4,5 & 4,7 & 5,1 & 5,0 & 5,0 & 5,3 & 5,2 & 5,4 \\
\hline
\end{tabular}

Fuente: World Bank (Health expenditure, public (\% of GDP). http://data.worldbank.org/indicator/SH.XPD.PUBL.ZS, accedido el Feb/2016). 
Tabla 3

Proporción de gastos privados, en relación al total de gastos con salud, y proporción de gastos con planes privados de salud, en relación al total de gastos privados. Argentina, Brasil, Chile y Colombia, 2000, 2005, 2010 y 2014.

\begin{tabular}{|c|c|c|c|c|c|c|c|c|}
\hline \multirow[t]{2}{*}{ Países } & \multicolumn{2}{|c|}{2000} & \multicolumn{2}{|c|}{2005} & \multicolumn{2}{|c|}{2010} & \multicolumn{2}{|c|}{2014} \\
\hline & Privado (\%) & Plan (\%) & Privado (\%) & Plan (\%) & Privado (\%) & Plan (\%) & Privado (\%) & Plan (\%) \\
\hline Argentina & 46,1 & 30,6 & 46,5 & 28,5 & 36,4 & 31,1 & 44,6 & 21,7 \\
\hline Brasil & 59,7 & 34,3 & 58,5 & 35,5 & 54,2 & 47,0 & 54,0 & 49,7 \\
\hline Chile & 71,7 & 34,5 & 62,6 & 32,7 & 52,7 & 36,1 & 50,5 & 37,6 \\
\hline Colombia & 20,7 & 41,0 & 25,8 & 34,1 & 26,4 & 32,6 & 24,9 & 38,3 \\
\hline
\end{tabular}

Fuente: Organización Mundial de la Salud (Global Health Observatory data repository. Health expenditure per capita, by country, $1995-2014$. http://apps.who.int/gho/data/node.main.484?lang=en, accedido el Feb/2016).

Tabla 4

Distribución de camas hospitalarias según naturaleza institucional, países seleccionados (Argentina 2012, Brasil 2015, Chile 2012 y Colombia 2016).

\begin{tabular}{|c|c|c|c|c|c|c|c|c|}
\hline \multirow[t]{3}{*}{ Países } & \multicolumn{8}{|c|}{ Camas hospitalarias } \\
\hline & \multicolumn{2}{|c|}{ Privadas } & \multicolumn{2}{|c|}{ Públicas } & \multicolumn{2}{|c|}{ Otras } & \multicolumn{2}{|c|}{ Total } \\
\hline & $\mathbf{n}$ & $\%$ & $\mathbf{n}$ & $\%$ & $\mathbf{n}$ & $\%$ & $\mathbf{n}$ & $\%$ \\
\hline Argentina & 59.549 & 42,1 & 81.885 & 57,9 & - & - & 141.434 & 100,0 \\
\hline Brasil & 197.923 & 71,1 & 77.117 & 27,7 & 3.362 * & 1,2 & 278.402 & 100,0 \\
\hline Chile & 6.292 & 15,0 & 27.776 & 69,0 & $6.083 * \star$ & 16,0 & 40.151 & 100,0 \\
\hline Colombia & 49.941 & 62,6 & 28.363 & 35,6 & $1.469 * * \star$ & 1,8 & 79.773 & 100,0 \\
\hline
\end{tabular}

* Otras: filantrópico y sindicato;

** Otras: centros psiquiátricos y geriátricos; institucionales -Fuerzas Armadas, cobre, universitarios-; mutuas;

*** Otras: instituciones prestadoras de salud que combinan recursos públicos y privados y son designadas como mixtas. Fuentes: elaboración propia, a partir de datos de Adecra+Cedim 39; Departamento de Informática do SUS

(CNES - Estabelecimentos por Tipo - Brasil. http://tabnet.datasus.gov.br/cgi/tabcgi.exe?cnes/cnv/estabbr.def, accedido el 05/Jul/2016); Clínicas de Chile A.G. 40; Ministerio de Salud y Protección Social de Colombia (Registro especial de prestadores de servicios de salud. https://prestadores.minsalud.gov.co/habilitacion/consultas/capacidad_departamental. aspx?pageTitle=Detallado+capacidad+instalada\&pageHlp, accedido el 11/Jul/2016).

Nótese, por lo tanto, que las estadísticas tampoco revelan la naturaleza más o menos pública de los sistemas de salud de los cuatro países, especialmente, en lo que se refiere a la inserción de las empresas de planes privados de salud. Pese a ello, es importante observar que tales informaciones son útiles para cuestionar las etiquetas de clasificación de los sistemas de salud. Se verifica que los países con componentes privados, claramente explicitados en la legislación, cuentan con una elevada proporción de financiación pública y que la capacidad instalada de camas predominante es privada, en Brasil y en Colombia, tampoco permite discernir el grado de privatización del sistema, puesto que el uso de la oferta no está necesariamente vinculado con la demanda privada.

\section{Consideraciones finales}

Una observación más atenta en la dinámica de la relación público/privada, considerando la existencia de múltiples límites, que son cada vez más difusos, puede contribuir a identificar aquellos elementos que estimulan y han estimulado los procesos de privatización como aquellos que han actuado como barreras a dichos procesos. El análisis comparado de algunos elementos específicos, como el análi- 
Tabla 5

Distribución temporal de la población que cuenta con un plan o seguro privado de salud. Argentina, Brasil, Chile y Colombia. $2000,2005,2010$ y 2014.

\begin{tabular}{|c|c|c|c|c|c|c|c|c|}
\hline \multirow[t]{2}{*}{ Países } & \multicolumn{2}{|c|}{2000} & \multicolumn{2}{|c|}{2005} & \multicolumn{2}{|c|}{2010} & \multicolumn{2}{|c|}{2014} \\
\hline & $\mathbf{n}$ & $\%$ & $\mathbf{n}$ & $\%$ & $\mathbf{n}$ & $\%$ & $\mathbf{n}$ & $\%$ \\
\hline Argentina * & $4.400 .000 * *$ & 12,1 & $5.489 .569 * \star \star$ & 18,0 & 6.222 .543 & 15,6 & - & - \\
\hline Brasil \# & 38.680 .406 & 24,5 & 43.202 .545 & 24,6 & 49.186 .989 & 25,9 & 56.088 .127 & 27,9 \\
\hline Chile \#\# & 3.092.195 & 20,0 & 2.660 .338 & 16,3 & 2.825 .618 & 16,5 & 3.206 .312 & 18,2 \\
\hline Colombia \#\#\# & - & 35,2 & - & 36,3 & 18.991 .790 & 47,5 & 21.284 .568 & 47,9 \\
\hline
\end{tabular}

* La población cubierta se refiere a los beneficiarios que cuentan con un plan de salud de una Empresa de Medicina Prepaga. Incluye a las personas que contratan de forma directa con la Empresa de Medicina Prepaga y a los denominados afiliados indirectos (que acceden a un plan de una Empresa de Medicina Prepaga, a través de una Obra Social);

** Los datos de 2000 corresponden a datos de 1999 y de una muestra realizada en el marco de la Encuesta de Desarrollo Social, mencionados en el trabajo de Acuña \& Chudnovsky 41;

*** Los datos de 2005 son calculados sobre una muestra población, realizada para la Encuesta Nacional de Utilización de Gastos y Servicios de Salud de 2005 42,43;

\# Los datos de Brasil se refieren a los años 1989, 2003 y 2008;

\#\# La población cubierta se refiere a aquellas personas que han elegido un plan de salud comercializado por una Institución de Salud y Seguridad Social (ISAPRE);

\#\#\# La población cubierta es la que se encuentra en el Régimen Contributivo y el porcentaje de la misma se realizó en base al total de la población afiliada al Sistema General de Seguridad Social en Salud (SGSSS). A diferencia de los tres países seleccionados, en los que la población de referencia es la población total o una muestra de la población total del país. Para los años 2000 y 2005 los datos fueron extraídos de Así Vamos em Salud Colombia (datos elaborados en base a Ministerios de Salud y Protección Social /BDUA Cuadro Afiliados BDUA y DANE. https://www.asivamosensalud. org/inidicadores/aseguramiento/grafica.ver/15, accedido el 05/Jul/2016) y para los años 2010 y 2014 de la Encuesta de Calidad de Vida, realizada por el Departamento Administrativo Nacional de Estadística 44,45. Población cubierta por el SGSSS sobre el total de la población en 2000: 58,8\%; 2005: 79,6\%; 2010: 47,5\%; 2014: 94,1\%.

Fuentes: elaboración propia en base a datos de Vargas Bustamante \& Méndez 30; Acuña \& Chudnovsky 41; Ministerio de Salud de la Nación 42; Departamento Administrativo Nacional de Estadística 44,45; Instituto Nacional de Estadística y Censos 46, Instituto Brasileiro de Geografía e Estatística 47, Fondo Nacional de Salud (https://www.fonasa.cl/portal_fonasa/site/artic/20140607/pags/20140607230746.html, accedido el 05/Jul/2016).

sis de gastos en salud, la propiedad de la infraestructura, la participación de las organizaciones que comercializan planes y seguros de salud en los sistema de salud, nos permitió identificar matices, contradicciones que se encuentran en los sistemas respecto a la relación público-privada, así como cuestionar el límite del uso de clasificaciones tradicionales sobre los mismos. Sin embargo, también pudimos observar la persistencia de cierta dificultad de algunos indicadores mencionados; como el gasto público y privado en salud, la distribución de camas según subsector; ya que no proyectan de manera adecuada la relación público-privada en los sistema de salud estudiados.

Por otra parte, entendemos la necesidad de superar la búsqueda de clasificaciones y de categorías bajo las cuales identificar sistemas de salud de países de la región, debido a los límites de las mismas para comprender las tensiones y contradicciones que existen en la dinámica de los sistemas de salud, como son las lecturas distorsionadas de los mismos. En este sentido creemos que una manera posible para aproximarnos a los mismos es a partir del análisis de políticas públicas específicamente seleccionadas, en función de su impacto en los procesos de privatización y el análisis de la trayectoria de las mismas, y no el análisis de sistemas de salud en su totalidad, tal como sugieren Marmor et al. 8 .

Por último, a pesar de las dificultades identificadas para la realización de estudios comparados, creemos que no son menores los aportes que los mismos son capaces de generar en términos de producción teórica y empírica para abrir nuevos debates, así como para poder revisar y cuestionar explicaciones previas. Se trata de buscar otros recorridos por territorios ya conocidos, pero que exigen nuevas preguntas y nuevos resultados. En definitiva, el propósito es poder contribuir a la elaboración de propuestas capaces de apuntar aspectos relevantes de las políticas de salud fuertemente sustentadas en una perspectiva integral, igualitaria y universal. 


\section{Colaboradores}

M. J. Luzuriaga participó de la redacción del artículo y elaboración de la estructura teórico-metodológica. L. Bahia colaboró en la redacción, estructura teórico-metodológica y revisión crítica del artículo.

\section{Agradecimientos}

Coordinación de Perfeccionamiento de Personal de Nivel Superior (Capes) y Consejo Nacional de Desarrollo Científico y Tecnológico (CNPq) por la financiación.

\section{Referencias}

1. Parsons T. Societies: evolutionary and comparative perspectives. New Jersey: PrenticeHall; 1966.

2. Bice TW, White KL. Factors related to the use of health services: an international comparative study. Med Care 1969; 7:124-33.

3. Roemer MI. Health care systems in world perspective. Ann Arbor: Health Administration Press; 1976.

4. Sidel VW. International comparisons of health services: how? Who? Why? Policy Stud J 1980; 9:300-7.

5. Evans DB, Tandon A, Murray CJL, Lauer JA. Comparative efficiency of national health systems: cross national econometric analysis. BMJ 2000; 323:307-10.

6. Marmor T, Wendt C. Conceptual frameworks for comparing healthcare politics and policy. Health Policy 2012; 107:11-20.

7. Marmor T, Okma KGH. Comparative studies and healthcare policy: learning and mislearning across borders. Clin Med (Lond) 2013; 13:487-91

8. Marmor T, Freeman R, Okma KGH. Comparative policy analysis and health care: an introduction. In: Marmor TR, Freeman R, Okma $\mathrm{KGH}$, editors. Comparative studies and politics of modern medical care. New Haven: Yale Press University; 2009. p. 1-23.

9. Okma KGH, Cheng T, Chinitz D, Crivelli L, Lim M, Maarse H, et al. Six countries, six health reform models? Health care reform in Chile, Israel, Singapore, Switzerland, Taiwan and the Netherlands. Journal of Comparative Policy Analysis: Research and Practice 2010; 12: 75-113.

10. Okma KGH, Crivelli L. Conclusions: debates, reforms and policy adjustments. In: Okma, KGH, Crivellli L, editores. Six countries, six reform models: the healthcare reform experience of Israel, the Netherlands, New Zealand, Singapore, Switzerland and Taiwan. Healthcare reforms "under the radar screen". Singapore: World Scientific Publishing; 2009. p. 205-13.
11. Mesa-Lago C. Social security in Latin America: pressure groups, stratification, and inequality. Pittsburgh: University of Pittsburgh Press; 1978.

12. Mesa-Lago C. Efectos de la crisis global sobre la seguridad social de salud y pensiones en América Latina. Santiago: Comisión Económica para Latin America y el Caribe; 2009. (Serie Políticas Sociales).

13. Fleury S, Belmartino S, Baris E. Reshaping health care in America Latina: a comparative analysis of health care reform in Argentina, Brazil, and Mexico. Ottawa: International Development Research Centre; 2000.

14. Fleury S. ¿Universal, dual o plural? Modelos y dilemas de la atención de la salud en América Latina: Chile, Brasil y Colombia. In: Molina CG, Núñez del Arco J, editores. Servicios de salud en América Latina y Asia. Washington DC: Banco Interamericano de Desarrollo; 2003. p. 3-37.

15. Labra ME. Políticas e saúde no Chile e no Brasil: contribuições para uma comparação. Ciênc Saúde Coletiva 2001; 6:361-76.

16. Almeida C. Reforma del sector salud y equidad en América Latina y el Caribe: conceptos, agenda, modelos y algunos resultados de implementación. Rev Gerenc Políticas Salud 2005; 4:6-60.

17. Hernández Álvarez M. La fragmentación de la salud en Colombia y Argentina: una comparación sociopolítica, 1880-1950. Bogotá: Universidad Nacional de Colombia; 2004.

18. Homedes N, Ugalde A, Privatización de los servicios de salud: las experiencias de Chile y Costa Rica. Gac Sanit 2002; 16:54-62.

19. Iriart C, Merhy E, Waitzkin H. La atención gerenciada en América Latina. Su papel en la reforma de los sistemas de salud. Buenos Aires: Instituto de Estudios y Formación-Central de los Trabajadores Argentinos; 1999. 
20. Londoño JL, Frenk J. Pluralismo estructurado: hacia un modelo innovador para la reforma de los sistemas de salud de América Latina. Washington DC: Banco Interamericano de Desarrollo; 1997. (Documento de Trabajo, 353).

21. Giovanella L, Feo O, Faria M, Tobar S. Sistemas de salud en Suramérica, desafíos para la universalidad, la integralidad y la equidad. Río de Janeiro: Instituto Suramericano de Gobierno en Salud; 2012.

22. Starr P. The meaning of privatization. Yale Law and Policy Review 1989; 6:6-41.

23. Maarse $H$. The privatization of health care in Europe: an eight-country analysis. J Health Polit Policy Law 2006; 31:981-1014.

24. Saltman RB. Melting public-private boundaries in European health systems. Eur J Public Health 2003; 13:24-9.

25. Bambra C. Worlds of welfare and the health care discrepancy. Soc Policy Soc 2005; 4:31-41.

26. Esping-Andersen G. As três economias políticas do Welfare State. Lua Nova: Revista de Cultura e Política 1991; 24:85-116.

27. Fox AM, Reich MR. The politics of universal health coverage in low and middle-income countries: a framework for evaluation and action. J Health Polit Policy Law 2015; 40: 1023-60.

28. Belmartino S, Bloch C. Desregulación/privatización: la relación entre financiación y provisión de los servicios en la reforma de seguridad social médica en la Argentina. Cuad Méd Soc (Ros.) 1998; 73:61-78.

29. Bahia L. O sistema de saúde brasileiro entre normas e fatos: universalização mitigada e estratificação subsidiada. Ciênc Saúde Coletiva 2009; 14:753-62.

30. Vargas Bustamante A, Méndez CA. Health care privatization in Latin America: compararing divergent privatization approaches in Chile, Colombia, and Mexico. J Health Polit Policy Law 2014; 39:841-86.

31. World Bank. Private voluntary health insurance: consumer protection and prudential regulation. Washington DC: World Bank; 2012.

32. Bahia L. Padrões e mudanças das relações público-privado: os planos e seguros saúde no Brasil [Tese de Doutorado]. Río de Janeiro: Escola Nacional de Saúde Pública, Fundação Oswaldo Cruz; 1999.

33. OECD Study on Private Health Insurance. Proposal for a taxonomy of health insurance. Paris: Organisation for Economic Co-operation and Development; 2004.

34. Kiil A. What characterises the privately insured in universal health care systems? A review of the empirical evidence. Health Policy 2012; 106:60-75
35. Belmartino S, Bloch C. El sistema de servicios de salud en Argentina. In: González Pérez GJ, Vega López MG, Romero Valle S, editores. Los sistemas de salud en Iberoamérica, de cara al siglo XXI. Jalisco: Organización Panamericana de la Salud; 2004. p. 11-32.

36. Belló M, Becerril-Montekio VM. Sistema de salud de Argentina. Salud Pública Méx 2011; 53 Suppl 2:S96-108.

37. Hoyos CEC. Salud y seguridad social: un breve comparativo de cinco países de América Latina. Bogotá: Friedrich Ebert Stiftung Colombia; 2012. (Análisis, 8).

38. Luzuriaga MJ. Regulación, medicina privada y ciudadanía: un estudio acerca de las denuncias presentadas ante la Subsecretaría de Defensa del Consumidor de la Nación sobre los seguros de salud de las empresas de medicina prepaga durante el periodo 2000-2008 [Disertación de Maestría]. Buenos Aires: Instituto de Salud Colectiva, Universidad Nacional de Lanús; 2012.

39. Adecra+Cedim. Las clínicas y sanatorios privados en la Argentina: su importancia económica y sanitaria. Buenos Aires: Adecra+Cedim; 2012. (Estudio Económico, 10).

40. Clínicas de Chile A.G. Dimensionamiento del sector de salud privado en Chile (cifras año 2012). Santiago: Clínicas de Chile A.G.; 2013.

41. Acuña C, Chudnovsky M. El sistema de salud en Argentina. San Andrés: Centro de Estudios para el Desarrollo Institucional, Fundación Gobierno y Sociedad; 2002. (Documento, 60).

42. Ministerio de Salud de la Nación. Encuesta Nacional de Gastos y Servicios de Salud. Buenos Aires: Ministerio de Salud de la Nación; 2005.

43. Maceira D. Sistema de salud en Argentina: la inequidad en la salud. In: Barten F, Flores W, Hardoy A, editores. Hacia un abordaje integral. Buenos Aires: IIED América Latina Publicaciones; 2008. p. 67-80.

44. Departamento Administrativo Nacional de Estadística. Encuesta Nacional de Calidad de Vida 2010. Bogotá: Departamento Administrativo Nacional de Estadística; 2011.

45. Departamento Administrativo Nacional de Estadística. Encuesta Nacional de Calidad de Vida 2015. Bogotá: Departamento Administrativo Nacional de Estadística; 2016.

46. Instituto Nacional de Estadísticas y Censos. Censo Nacional de Población y Vivienda de 2010. Buenos Aires: Instituto Nacional de Estadísticas y Censos; 2010

47. Instituto Brasileiro de Geografia e Estatística. Pesquisa Nacional por Amostra de Domicílios: acesso e utilização de serviços de saúde. Rio de Janeiro: Instituto Brasileiro de Geografia e Estatística; 2008. 


\section{Abstract}

The aim was to conduct a literature review on health systems and comparative studies of health policies and systems to identify the leading contributions to the analysis of privatization, especially the role of health maintenance organizations and health insurance companies in national health systems in Latin America. The review focused on analyzing the adequacy of theoretical and methodological perspectives or proposals and their indicators to measure the private health sector's participation and interaction with national health systems. We thus seek to problematize the adequacy, limits, and potentialities of such perspectives for investigating the privatization processes in Latin American health systems. The comparative analysis of some specific elements such as health expenditures, installed capacity, and population covered by health plans and health insurance allowed us to identify contradictions in the systems regarding public-private relations, as well as to question the use of traditional classifications for defining various health systems in the region. Our challenge was to break with linear, non-contradictory explanations for analyzing health systems. This perspective requires questioning categories and typologies that have been developed and used repeatedly in order to discuss and rethink to move forward with a more rigorous understanding of our realities, as well as to encourage new debates and explanations.

Privatization; Health Maintenance Organizations; Health Systems; Health Policy; Latin America

\section{Resumo}

O estudo teve como objetivo fazer uma revisão bibliográfica sobre os sistemas de saúde e estudos comparados em politicas e sistemas de saúde para identificar os principais aportes dos mesmos na análise da privatização, especialmente, no papel das organizações que comercializam planos e seguros de saúde nos sistemas nacionais de saúde em países da América Latina. A revisão foi focada em analisar a adequação das perspectivas ou propostas teórico-metodológicas de seus indicadores para dimensionar a participação do sector privado da saúde, e sua interação com os sistemas nacionais de saúde. Deste modo, procuramos problematizar a adequação, os limites e as potencialidades dos mesmos, estudando os processos de privatização nos sistemas de saúde de países da América Latina. As análises comparadas de alguns elementos específicos, como as análises de custos em saúde, a capacidade instalada, a população coberta com planos e seguros de saúde, nos permitiu identificar as vinculações e contradições existentes nos sistemas, ao respeito das relações com o sistema público- privado, além de questionarmos o uso das classificações tradicionais com as quais foram definidos os diferentes sistemas de saúde em nossa região. Nosso desafio foi romper com algumas explicações lineares o carentes de contradições sobre as análises dos sistemas de saúde. Desde essa perspectiva é necessário questionar as categorias ou tipologias que foram desenvolvidas e usadas de forma recorrente, com o fim de discuti-las e repensá-las a fim de avançar numa compreensão mais rigorosa das nossas realidades, assim como estimular novos debates e explicações sobre esta questão.

Privatização; Sistemas Pré-Pagos de Saúde; Sistemas de Saúde; Política de Saúde; América Latina
Recibido el 20/Jul/2016

Versión final presentada el 24/Nov/2016

Aprobado el 14/Dic/2016 